\title{
Transesophageal versus transcranial motor evoked potentials to monitor spinal cord ischemia
}

\author{
Kazumasa Tsuda, MD, ${ }^{\mathrm{a}}$ Norihiko Shiiya, MD, PhD, ${ }^{\mathrm{a}}$ Daisuke Takahashi, MD, ${ }^{\mathrm{a}}$ \\ Kazuhiro Ohkura, MD, $\mathrm{PhD},{ }^{\mathrm{a}}$ Katsushi Yamashita, $\mathrm{MD}, \mathrm{PhD},{ }^{\mathrm{a}}$ Yumi Kando, $\mathrm{MD},{ }^{\mathrm{a}}$ and \\ Yoshifumi Arai, MD, $\mathrm{PhD}^{\mathrm{b}}$
}

\begin{abstract}
Objectives: We have previously reported that transesophageal motor evoked potential is feasible and more stable than transcranial motor evoked potential. This study aimed to investigate the efficacy of transesophageal motor evoked potential to monitor spinal cord ischemia.
\end{abstract}

Methods: Transesophageal and transcranial motor evoked potentials were recorded in 13 anesthetized dogs at the bilateral forelimbs, anal sphincters, and hindlimbs. Spinal cord ischemia was induced by aortic balloon occlusion at the 8th to 10th thoracic vertebra level. In the 12 animals with motor evoked potential disappearance, occlusion was maintained for 10 minutes $(\mathrm{n}=6)$ or 40 minutes $(\mathrm{n}=6)$ after motor evoked potential disappearance. Neurologic function was evaluated by Tarlov score at 24 and 48 hours postoperatively.

Results: Time to disappearance of bilateral motor evoked potentials was quicker in transesophageal motor evoked potentials than in transcranial motor evoked potentials at anal sphincters $(6.9 \pm 3.1$ minutes vs $8.3 \pm 3.4$ minutes, $P=.02)$ and hindlimbs $(5.7 \pm 1.9$ minutes vs $7.1 \pm 2.7$ minutes, $P=.008)$. Hindlimb function was normal in all dogs in the 10-minute occlusion group, and motor evoked potentials recovery ( $>75 \%$ on both sides) after reperfusion was quicker in transesophageal motor evoked potentials than transcranial motor evoked potentials at hindlimbs $(14.8 \pm 5.6$ minutes vs $24.7 \pm 8.2$ minutes, $P=.001)$. At anal sphincters, transesophageal motor evoked potentials always reappeared $(>25 \%)$, but transcranial motor evoked potentials did not in 3 of 6 dogs. In the 40-minute occlusion group, hindlimb motor evoked potentials did not reappear in 4 dogs with paraplegia. Among the 2 remaining dogs, 1 with paraparesis (Tarlov 3 ) showed delayed recovery $(>75 \%)$ of hindlimb motor evoked potentials without reappearance of anal sphincter motor evoked potentials. In another dog with spastic paraplegia, transesophageal motor evoked potentials from the hindlimbs remained less than $20 \%$, whereas transcranial motor evoked potentials showed recovery $(>75 \%)$.

Conclusions: Transesophageal motor evoked potentials may be superior to transcranial motor evoked potentials in terms of quicker response to spinal cord ischemia and better prognostic value. (J Thorac Cardiovasc Surg 2016;151:509-17)

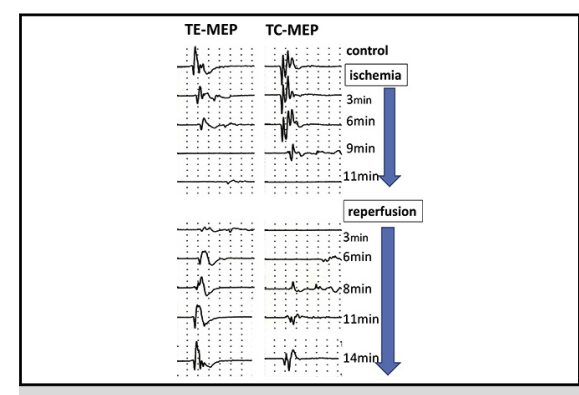

Response to ischemia/reperfusion was quicker in TEMEP than in TC-MEP.

Central Message

TE-MEP is superior to TC-MEP in terms of quicker response to spinal cord ischemia and better prognostic value.

\section{Perspective}

This study addresses the low specificity of TCMEP to monitor spinal cord ischemia during thoracic aortic surgery, which is largely due to its instability. The results suggest that TEMEP, which we have shown is more stable than TC-MEP, is superior to TC-MEP in terms of quicker response to ischemia and better prognostic value. It will provide technical ease and improved accuracy in the event of clinical application.

See Editorial Commentary page 518.

\footnotetext{
From the ${ }^{\mathrm{a}}$ First Department of Surgery, Hamamatsu University School of Medicine, Higashi-ku, Hamamatsu, Shizuoka, Japan; and ${ }^{\mathrm{b}}$ Department of Pathology, Seirei Hamamatsu Hospital, Naka-ku, Hamamatsu, Shizuoka, Japan.

This work was supported by the Grants-In-Aid for Scientific Research of the Japanese Ministry of Education, Culture, Sports, Science, and Technology (Grant 24592056)

Read at the 95th Annual Meeting of The American Association for Thoracic Surgery, Seattle, Washington, April 25-29, 2015.
}

Received for publication April 13, 2015; revisions received Aug 5, 2015; accepted for publication Aug 22, 2015; available ahead of print Oct 13, 2015.

Address for reprints: Norihiko Shiiya, MD, PhD, First Department of Surgery, Hamamatsu University School of Medicine, 1-20-1 Handayama, Higashi-ku, Hamamatsu, Shizuoka 431-3192, Japan (E-mail: shiyanor@hama-med.ac.jp). $0022-5223 / \$ 36.00$

Copyright (C) 2016 by The American Association for Thoracic Surgery http://dx.doi.org/10.1016/j.jtcvs.2015.08.120 


\section{Abbreviations and Acronyms \\ MEP $=$ motor evoked potential \\ $\mathrm{TE}=$ transesophageal \\ $\mathrm{TC}=$ transcranial}

Myogenic transcranial (TC) motor evoked potentials (MEPs) have been widely used to monitor spinal cord ischemia during aortic surgery as a component of a multidisciplinary spinal cord protection strategy. ${ }^{1,2}$ This is because of the quickness in response and high sensitivity compared with other monitoring methods, which enables surgeons to react promptly to spinal cord ischemia during surgery. ${ }^{3,4}$ On the other hand, instability and amplitude fluctuation of TC-MEP have been a clinical problem, which results in the low specificity when the cutoff value is set high. This may explain why the cutoff amplitude of the evoked potentials varies considerably from $25 \%$ to $75 \%$ of baseline among the investigators. ${ }^{5,6}$ In addition, recovery of TC-MEP after transient loss does not guarantee prevention from neurologic dysfunction, ${ }^{7}$ which makes the prognostic value of TCMEP low. ${ }^{7,8}$

We have previously reported that transesophageal (TE) electrical stimulation of the spinal cord can safely and easily elicit myogenic MEPs by conventional equipment for TC-MEP. ${ }^{9}$ We found that stimulation intensity was always supra-maximal for TE-MEP, which may be desirable to avoid amplitude fading of myogenic potentials, and interindividual variability is small. This made us hypothesize that specificity and accuracy to spinal cord ischemia may be improved by TE stimulation if its response is at least as quick and sensitive as that of TCMEP.

The concept of monitoring myogenic potentials elicited by direct stimulation of the spinal cord has been proposed by Mochida and colleagues ${ }^{10}$ by the use of epidural electrodes for stimulation. Although they have reported that it can promptly detect spinal cord ischemia induced by thoracic aortic crossclamping, it has rarely been used clinically in aortic surgery because of the need for an epidural electrode. Because our technique of TE spinal cord stimulation differs considerably from epidural stimulation, myogenic response may be completely different. The purpose of the present study is to investigate whether or not response of TE-MEP to spinal cord ischemia is as prompt and accurate as that of TC-MEP in a canine model of spinal cord ischemia.

\section{MATERIALS AND METHODS}

Thirteen adult beagle dogs (weight, 12.7-20.0 kg) were used. All animals received humane care in compliance with the "Guide for the Care and Use of Laboratory Animals" published by the National Institutes of
Health (publication 85-32, revised 1985). The institutional ethics committee on the use and care of animals approved the experimental protocol (No. 2013014).

\section{Experimental Settings}

Methods of anesthesia and instrumentation have been reported. ${ }^{9}$ Briefly, the animals were anesthetized with intravenous infusion of propofol $(12-24 \mathrm{mg} / \mathrm{kg} / \mathrm{h})$ and remifentanil $(12-24 \mu \mathrm{g} / \mathrm{kg} / \mathrm{h})$ without muscle relaxant, and were intubated and maintained on mechanical ventilation. For MEP recording, a Neuropak MEB-2200 system (Nihon Kohden, Tokyo, Japan) was used for data acquisition, processing, and analysis, with a SEN-4100 equipment for electrical stimulation (Nihon Kohden, Tokyo, Japan). For TC stimulation of the brain motor area, a cathode was placed on the $\mathrm{C} 4$ position and an anode on the $\mathrm{C} 3$ position of the international 10-20 system. TE stimulation of the spinal cord was performed between the handmade esophageal luminal surface electrode (cathode) and a nuchal subcutaneous needle electrode (anode) that was set at about the fourth to fifth thoracic spine level as a target. A train of 5 rectangular pulses was used with a $2.0-\mathrm{ms}$ interstimulus interval at $500 \mathrm{~V}$ with a 0.05 -ms pulse width. MEPs were recorded at the bilateral abductor pollicis brevis muscles, anterior tibial muscles, and external anal sphincter muscles. Needle electrodes were used to record compound muscle action potentials from the limbs, and a plug-type electrode (a special order product, Nihon Kohden) was used to record external anal sphincter muscle potentials.

\section{Experimental Model}

Spinal cord ischemia was induced by inflating an aortic occlusion balloon catheter (Reliant, Medtronic Inc, Minn) at the eighth to tenth thoracic vertebra level. The balloon was introduced through a femoral artery under fluoroscopic control after $100 \mathrm{U} / \mathrm{kg}$ heparin was administered (Figure 1). To confirm aortic occlusion, arterial blood pressure was continuously recorded at the contralateral femoral artery and left common carotid artery. No attempt was made to control proximal blood pressure during aortic occlusion.

\section{Experimental Protocol}

Before thoracic aortic occlusion, the terminal aorta was balloonoccluded in each animal for 30 minutes to evaluate the influence of lower-limb ischemia on MEPs. Then the balloon was deflated and advanced to the target level of the descending thoracic aorta. After an interval of 15 minutes, the balloon was inflated and the descending thoracic aorta was occluded. TC- and TE-MEP were recorded every 2 or 3 minutes, and time from aortic occlusion to disappearance of waveforms was measured on each recording site. MEP did not disappear by descending aortic occlusion in $1 \mathrm{dog}$, and this animal was not used for subsequent experiment. In the remaining 12 animals, balloon occlusion was maintained for an additional 10 minutes $(n=6)$ or 40 minutes $(\mathrm{n}=6)$ after disappearance of all MEPs. Time to MEP disappearance ranged from 4 to 18 minutes, so that total duration of thoracic aortic occlusion was 15 to 21 minutes in the 10-minute occlusion group and 44 to 58 minutes in the 40-minute occlusion group. Then balloon occlusion was released, and time to reappearance of waveform on both MEPs at the anal sphincter and hindlimbs was measured up to 60 minutes after reperfusion.

The animals were allowed to recover with all catheters removed, arteries repaired, and wounds closed. Neurologic function was evaluated by a person who was blinded to the monitor results according to the modified Tarlov classification ( 0 : no movement of hindlimbs, 1: perceptible movement of the joints of the hindlimbs, 2: good movement but unable to stand, 3: able to stand and walk, 4: complete recovery) at the completion of and 24 and 48 hours after the procedure. Animals with paralysis were 


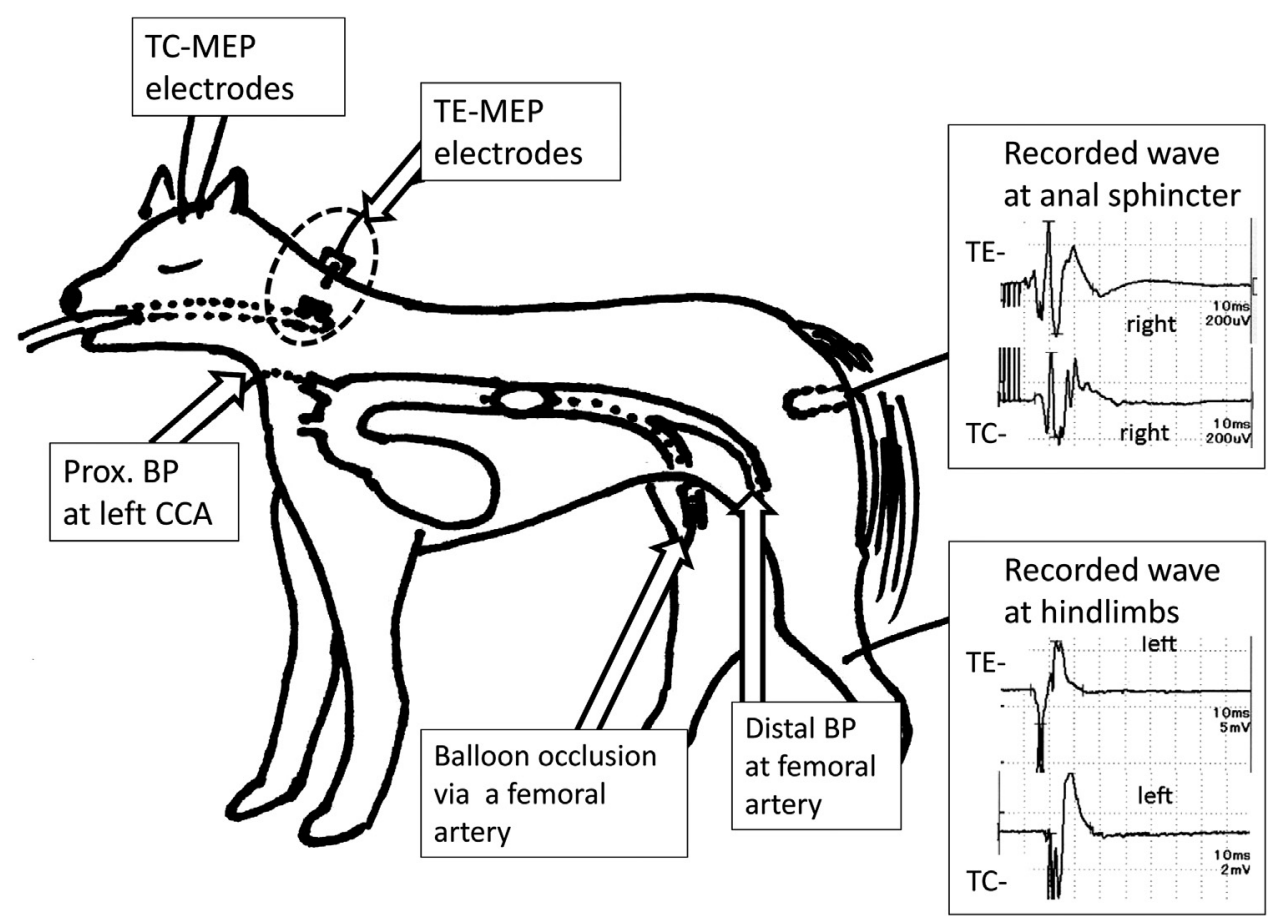

FIGURE 1. Schematic drawing of the experimental model. Typical waveforms of myogenic MEPs from anal sphincters and hindlimbs without spinal cord ischemia are described together. $T C$, Transcranial; $M E P$, motor evoked potential; $T E$, transesophageal; Prox, proximal; $B P$, blood pressure; $C C A$, common carotid artery.

killed at 48 postoperative hours, and the explanted spinal cords were histopathologically evaluated.

\section{Statistical Analysis}

Each value was expressed as the mean \pm standard deviation. Statistical analyses were performed using IBM SPSS Statistics software (version 21, IBM Japan, Tokyo, Japan). For comparisons of the baseline amplitudes and latencies at baseline or after recovery, and for comparisons of the time to amplitude change, variance components analysis using the univariate general linear model was performed, with the stimulation methods (TE vs TC) and recording sides (right vs left) as fixed factors and individual animals as a random factor.

\section{RESULTS}

At baseline, amplitudes were similar between TE-MEP and TC-MEP, and latencies were shorter in TE-MEP than in TC-MEP (Table 1). Terminal aortic occlusion for $30 \mathrm{mi}-$ nutes did not result in MEP change in all 13 animals. Thoracic aortic occlusion resulted in MEP disappearance in 12 dogs. Time to amplitudes decrease to $75 \%, 50 \%$, and $25 \%$ of control was significantly shorter in TE-MEP than in TC-MEP at the hindlimbs, but the difference was not statistically significant at the anal sphincters. Time to MEP disappearance was significantly shorter in TE-MEP than in TC-MEP at both the anal sphincters and the hindlimbs (Table 2). There was no difference between the right and left sides. In the remaining $1 \mathrm{dog}$, amplitudes of MEPs decreased to less than $40 \%$ of control at all recording points (TE-MEP: anal sphincter left $17 \%$, right $27 \%$, hindlimb left $40 \%$, right $27 \%$; TC-MEP: anal sphincter left $28 \%$, right $29 \%$, hindlimb left $37 \%$, right $16 \%$ of control), but they did not disappear completely after 30 minutes of aortic

TABLE 1. Baseline amplitudes and latencies of motor evoked potentials $(N=13)$

\begin{tabular}{|c|c|c|c|c|c|c|c|c|c|c|}
\hline & \multicolumn{5}{|c|}{ Anal sphincters } & \multicolumn{5}{|c|}{ Hindlimbs } \\
\hline & \multicolumn{2}{|c|}{ TE-MEP } & \multicolumn{2}{|c|}{ TC-MEP } & & \multicolumn{2}{|c|}{ TE-MEP } & \multicolumn{2}{|c|}{ TC-MEP } & \\
\hline & Left & Right & Left & Right & & Left & Right & Left & Right & \\
\hline \multirow[t]{2}{*}{ Amplitudes } & \multicolumn{4}{|c|}{$(\mu \mathrm{V})$} & \multicolumn{6}{|c|}{$(\mathrm{mV})$} \\
\hline & $302 \pm 217$ & $317 \pm 247$ & $262 \pm 224$ & $280 \pm 229$ & & $8.5 \pm 2.8$ & $8.4 \pm 2.6$ & $7.3 \pm 3.0$ & $7.8 \pm 2.9$ & \\
\hline Latencies (ms) & $12.9 \pm 2.6$ & $14.0 \pm 2.4$ & $16.0 \pm 2.6$ & $17.4 \pm 3.1$ & $* 1$ & $15.5 \pm 2.4$ & $15.8 \pm 2.8$ & $18.1 \pm 1.8$ & $18.6 \pm 2.6$ & $* 2$ \\
\hline
\end{tabular}

TE, Transesophageal; $M E P$, motor evoked potential; $T C$, transcranial. $* 1,2: P=.001$ (TE vs TC). 
TABLE 2. Time to amplitude reduction during aortic balloon occlusion $(\mathbf{N}=12)$

\begin{tabular}{|c|c|c|c|c|c|c|c|c|c|c|}
\hline & \multicolumn{5}{|c|}{ Anal sphincters } & \multicolumn{5}{|c|}{ Hindlimbs } \\
\hline & \multicolumn{2}{|c|}{ TE-MEP } & \multicolumn{2}{|c|}{ TC-MEP } & & \multicolumn{2}{|c|}{ TE-MEP } & \multicolumn{2}{|c|}{ TC-MEP } & \\
\hline & Left & Right & Left & Right & & Left & Right & Left & Right & \\
\hline Time to $<75 \%$ & $3.3 \pm 1.5$ & $3.2 \pm 1.1$ & $3.9 \pm 2.7$ & $3.1 \pm 1.2$ & & $4.2 \pm 2.0$ & $3.4 \pm 1.1$ & $4.4 \pm 1.2$ & $4.8 \pm 1.9$ & $* 1$ \\
\hline Time to $<50 \%$ & $3.8 \pm 1.4$ & $4.1 \pm 1.8$ & $5.8 \pm 4.1$ & $4.3 \pm 2.7$ & & $4.4 \pm 1.9$ & $3.9 \pm 0.9$ & $6.0 \pm 2.0$ & $5.8 \pm 2.2$ & $* 2$ \\
\hline Time to $<25 \%$ & $6.0 \pm 2.7$ & $6.0 \pm 2.9$ & $6.8 \pm 4.2$ & $6.9 \pm 3.5$ & & $5.2 \pm 2.2$ & $5.5 \pm 1.9$ & $7.0 \pm 2.8$ & $6.8 \pm 2.4$ & $* 3$ \\
\hline Time to disappearance & $6.8 \pm 3.5$ & $7.1 \pm 3.3$ & $8.3 \pm 3.5$ & $8.4 \pm 3.6$ & $* 4$ & $5.7 \pm 1.9$ & $5.7 \pm 1.9$ & $7.3 \pm 2.8$ & $6.9 \pm 2.5$ & $* 5$ \\
\hline
\end{tabular}

Expressed in minutes. $T E$, Transesophageal; $M E P$, motor evoked potential; $T C$, transcranial. *1: $P=.03$ (TE vs TC). *2: $P=.01$ (TE vs TC). *3:P=.003 (TE vs TC). *4: $P=.02$ (TE vs TC). *5: $P=.008$ (TE vs TC).

occlusion. After releasing the aortic occlusion, all MEPs showed complete recovery, and the animal was neurologically intact. This dog was excluded from the subsequent experiment.

Blood pressures before and during thoracic aortic occlusion were continuously recorded at the left common carotid artery and the femoral artery. At the carotid artery, they were $149 \pm 22 / 87 \pm 15 / 103 \pm 16$ (expressed as systolic/diastolic/mean in $\mathrm{mmHg}$ ) before occlusion and $176 \pm 26 / 111 \pm 23 / 130 \pm 24$ during occlusion in the 13 animals. At the femoral artery, they were $30 \pm 5$ / $23 \pm 8 / 25 \pm 6$ during aortic occlusion. In the excluded dog, proximal blood pressures before and during aortic occlusion were 178/106/126 and 222/153/172, and distal blood pressure during occlusion was $32 / 25 / 27$. It is noteworthy that proximal blood pressure during aortic occlusion was almost equal to mean \pm 2 standard deviation of the 13 dogs.

In the 10-minute occlusion group $(\mathrm{n}=6)$, amplitude of both TE-MEP and TC-MEP from the hindlimbs showed recovery to more than $75 \%$ of control after reperfusion in all animals. Time to recovery was significantly shorter in TE-MEP than in TC-MEP (Table 3). This was also true if the cutoff was set at $50 \%$ or $25 \%$ of control (Table 3). When the amplitude recovered to $75 \%$ of control, latency remained prolonged compared with the baseline, which was significantly longer in TC-MEP than in TE-MEP (TE-MEP: left $107 \% \pm 9 \%$, right $102 \% \pm 9 \%$, TC-MEP: left $113 \% \pm 9 \%$, right $112 \% \pm 8 \%$ of baseline, $P=.008$ ). Representative change in MEP waveform from hindlimbs was shown in Figure 2. Recovery of anal sphincter MEPs, on the other hand, was incomplete, and it was inconsistent for TCMEPs. All the 6 animals showed recovery to more than $25 \%$ of control within 30 minutes by TE stimulation, whereas only 3 dogs showed recovery to more than $25 \%$ of control by TC stimulation. Recovery to more than $75 \%$ was achieved in 2 of 6 animals in anal sphincter TE-MEP, whereas it was never achieved in anal sphincter TC-MEP. There was no spinal cord dysfunction in these 6 animals.

In the 40-minute occlusion group $(n=6)$, both TE- and TC-MEPs from hindlimbs and anal sphincters did not reappear after reperfusion in 4 dogs, and they were completely paraplegic (Tarlov grade 0) throughout the postoperative period. In the 2 remaining dogs, $1(\operatorname{dog} 5)$ showed delayed and inconsistent recovery of both TE-MEP and TC-MEP at hindlimbs and no recovery at anal sphincters (Table 4). This dog developed immediate paraparesis (Tarlov grade 3). Another $\operatorname{dog}(\operatorname{dog} 6)$ demonstrated relatively slow disappearance of TC-MEP (18 minutes at bilateral anal sphincters, left 13 minutes, and right 10 minutes at hindlimb) and TE-MEP at bilateral anal sphincters (16 minutes), but quick disappearance of TE-MEP at bilateral hindlimbs (2 minutes). In this dog, TE-MEP from hindlimbs remained less than $20 \%$ after reperfusion, whereas TCMEP at hindlimbs showed complete recovery $(>75 \%)$ at 30 minutes and thereafter (Table 4). At anal sphincters, both TE-MEP and TC-MEP showed delayed partial $(>50 \%)$ recovery (Table 4$)$. This dog developed progressive spastic paraplegia that was Tarlov 3 immediately after the experiment and became Tarlov 0 at 48 hours. Waveforms of TC-MEP and TE-MEP from hindlimbs in these 2 dogs are shown in Figure 2.

TABLE 3. Time to amplitude recovery at hindlimbs after releasing balloon occlusion in the 10-minute occlusion group $(N=6)$

\begin{tabular}{|c|c|c|c|c|c|}
\hline & \multicolumn{2}{|c|}{ TE-MEP } & \multicolumn{2}{|c|}{ TC-MEP } & \\
\hline & Left & Right & Left & Right & \\
\hline Time to $>25 \%$ & $9.5 \pm 4.3(4$ to 14$)$ & $8.5 \pm 3.4(5$ to 14$)$ & $15.2 \pm 8.8(5$ to 28$)$ & $16.7 \pm 10.4(5$ to 33$)$ & $* 1$ \\
\hline Time to $>50 \%$ & $10.8 \pm 4.6(5$ to 18$)$ & $11.0 \pm 4.0(5$ to 15$)$ & $18.3 \pm 8.6(12$ to 33$)$ & $18.0 \pm 9.7(5$ to 33$)$ & $* 2$ \\
\hline Time to $>75 \%$ & $15.2 \pm 6.9(12$ to 28$)$ & $14.3 \pm 6.5(5$ to 24$)$ & $24.2 \pm 7.0(18$ to 38$)$ & $25.3 \pm 9.6(12$ to 38$)$ & $* 3$ \\
\hline
\end{tabular}

Expressed in minutes. TE, Transesophageal; $M E P$, motor evoked potential; $T C$, transcranial. *1: $P=.002$ (TE vs TC). *2: $P=.001$ (TE vs TC). *3: $P=.001$ (TE vs TC). 


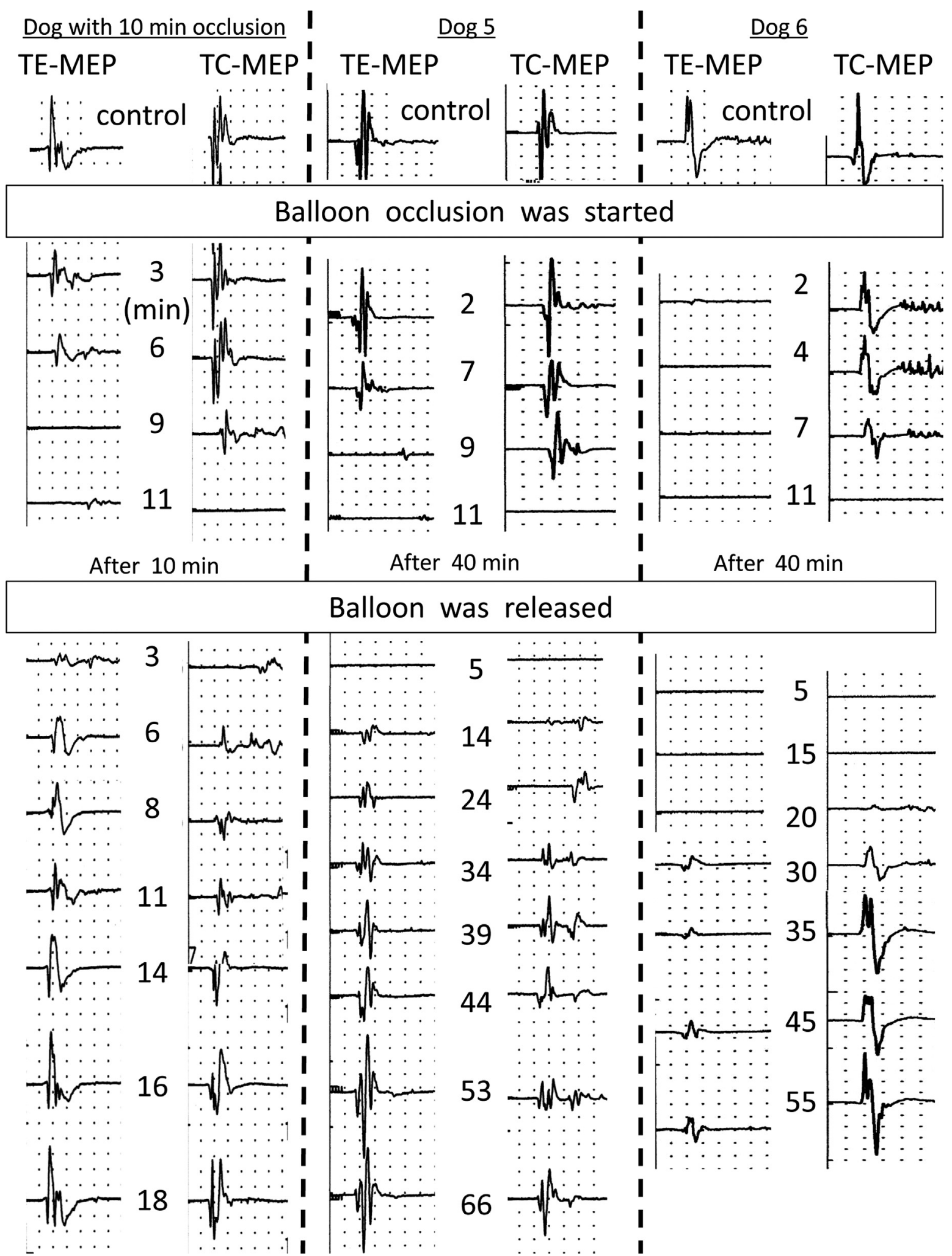

FIGURE 2. Change in the waveforms of hindlimb myogenic MEPs during and after thoracic aortic occlusion. Left: a dog in the 10-minute occlusion group. Middle: dog 5 that developed paraparesis. Right: dog 6 that developed spastic paraplegia. TE, Transesophageal; MEP, motor evoked potential; $T C$, transcranial. 
TABLE 4. Time to amplitude recovery after releasing balloon occlusion in the 2 dogs that developed paraparesis $(\operatorname{dog} 5)$ or spastic paraplegia $(\operatorname{dog} 6)$ after 40 minutes of occlusion

\begin{tabular}{|c|c|c|c|c|c|c|c|c|}
\hline & \multicolumn{4}{|c|}{ Anal sphincters } & \multicolumn{4}{|c|}{ Hindlimbs } \\
\hline & \multicolumn{2}{|c|}{ TE-MEP } & \multicolumn{2}{|c|}{ TC-MEP } & \multicolumn{2}{|c|}{ TE-MEP } & \multicolumn{2}{|c|}{ TC-MEP } \\
\hline & Left & Right & Left & Right & Left & Right & Left & Righ \\
\hline \multicolumn{9}{|l|}{ Dog 5} \\
\hline Time to $>25 \%$ & - & - & - & - & 24 & 24 & 18 & 34 \\
\hline Time to $>50 \%$ & - & - & - & - & 29 & 39 & 44 & 60 \\
\hline Time to $>75 \%$ & - & - & - & - & 34 & 53 & 53 & - \\
\hline \multicolumn{9}{|l|}{$\operatorname{Dog} 6$} \\
\hline Time to $>25 \%$ & 45 & 35 & 30 & 20 & - & - & 20 & 30 \\
\hline Time to $>50 \%$ & 50 & 50 & 30 & 30 & - & - & 30 & 35 \\
\hline Time to $>75 \%$ & - & - & - & - & - & - & 30 & 35 \\
\hline
\end{tabular}

Expressed in minutes. TE, Transesophageal; $M E P$, motor evoked potential; $T C$, transcranial.

\section{Histopathology}

Light micrographs of dogs 4 (immediate paraplegia Tarlov 0), 5, and 6 are shown in Figure 3. In dog 4, an ill-defined border between the white and gray matters indicated severe damage of the spinal cord at the lower thoracic vertebra level. In the lumber anterior horn, there were large neuronal cells with red tinctured nucleus that suggested necrotic cell death and infiltration of mononuclear cells in the surrounding gray matter.
These findings agreed with severe ischemic spinal cord dysfunction. In $\operatorname{dog} 5$, thoracic spinal tract injury of the white matter was not evident, but shrunk neuronal cells could be seen among the large neuronal cells in the lumber anterior horn, which were compatible with selective motor neuron death. In dog 6, damage of the white matter, especially around the lateral column, was evident at the thoracic spinal cord. In the lumber anterior horn, on the other hand, there were large neuronal cells with a normal-appearing nucleus. These findings suggests predominant thoracic spinal tract injury with minimum motor neuron damage at the lumbar spinal cord.

\section{DISCUSSION}

The present study showed that the response to spinal cord ischemia was quicker in TE-MEP than in TC-MEP. In the animals without motor dysfunction of hindlimbs, amplitude recovery after reperfusion was also quicker in TE-MEP than TC-MEP at hindlimbs, and recovery to more than $75 \%$ of baseline was achieved within 30 minutes in TE-MEP and 40 minutes in TC-MEP. On the other hand, recovery of anal sphincter MEP was incomplete in these dogs, but reappearance of TE-MEP was always achieved. Except for anal sphincter TC-MEP, loss of MEP without recovery after reperfusion was associated
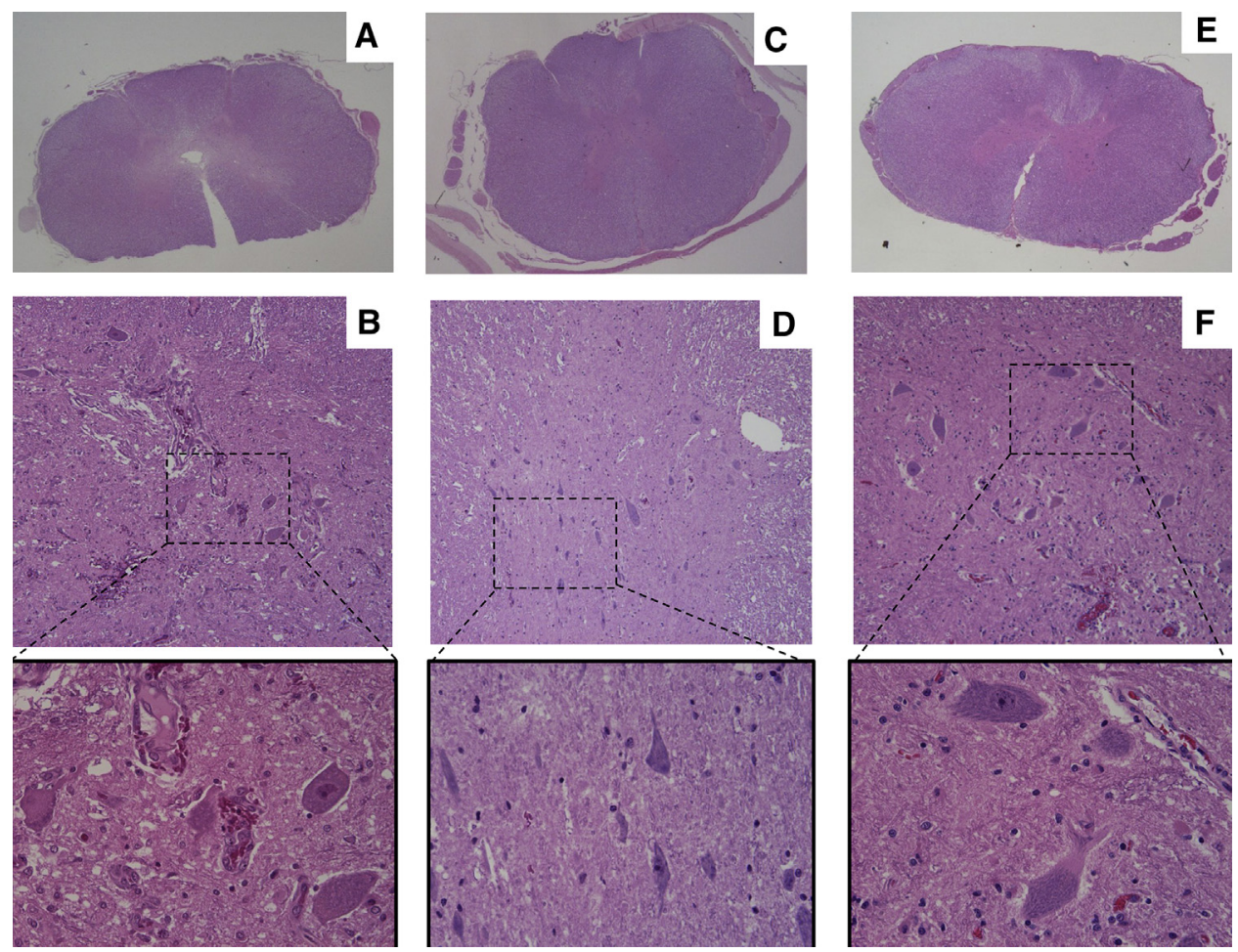

FIGURE 3. Light micrographs of the lower thoracic spinal cord $(A, C, E$, hematoxylin-eosin, $\times 10)$ and anterior horn of the proximal lumbar spinal cord (B, D, F, hematoxylin-eosin, upper bank; $\times 100$, lower bank; $\times 400$ ). A and B were taken from $\operatorname{dog} 4, \mathrm{C}$ and $\mathrm{D}$ were taken from $\operatorname{dog} 5$, and $\mathrm{E}$ and $\mathrm{F}$ were taken from $\operatorname{dog} 6$. 
with immediate paraplegia, but recovery of MEP did not always mean that the animals were neurologically intact, regardless of the stimulation method. However, only TEMEP may predict incomplete paralysis by delayed recovery of hindlimb amplitudes with persistent loss of anal sphincter potentials. Spastic paraplegia also may be predicted only by TE-MEP.

Incidence of spinal cord injury in a canine model of simple descending aortic crossclamping varies considerably among the reports. This is due to the presence of rich collateral blood pathway in dogs. Sugawara and colleagues ${ }^{11}$ have reported that 4 of 6 dogs developed spinal cord injury by 60 minutes of simple aortic crossclamping at the proximal descending aorta, whereas all the 6 animals were paraplegic after 40 minutes when proximal blood pressure was controlled to $80 \mathrm{~mm} \mathrm{Hg} .{ }^{11}$ We did not control proximal blood pressure, and both proximal and distal blood pressures were similar to those of Sugawara and colleagues' 60-minute occlusion model. Thus, the incidence of MEP disappearance (12/13) was higher than expected. This may be related to the use of balloon occlusion at the low descending aorta, but the exact mechanism remains to be investigated. On the other hand, $100 \%$ incidence of spinal cord injury in the 40-minute occlusion group was not surprising, because incidence of spinal cord injury in the dog model of descending aortic occlusion depends on the amount of collateral blood flow, and loss of MEP indicates that spinal cords were in severe ischemia in these animals.

The quickness in response to ischemia/reperfusion at the hindlimbs is the advantage of TE-MEP over TCMEP because tolerable duration of ischemia is relatively short in the spinal cord. This will enable surgeons to apply protective procedures and judge their effect more promptly. The reason for this quickness remains to be elucidated, but 2 mechanisms may be involved. One is the difference in stimulation condition between TE-MEP and TC-MEP, and another is the difference in response to spinal tract ischemia.

We have previously reported that TE stimulation can be performed by supra-maximal intensity, whereas TC stimulation intensity is usually supra-threshold. ${ }^{9}$ This is due to the safety issue of brain electrical stimulation and difficulty in correctly positioning TC stimulation electrodes. It is known that response of alpha motor neurons to excitatory post-synaptic potentials is nonlinear, and an input exceeding a firing threshold produces a full response and anything below it produces no response. Thus, amplitude of compound muscle action potentials should reflect the number of responding alpha motor neurons. ${ }^{12}$ This means that TE stimulation by supra-maximal intensity activates alpha motor neurons as many as possible, whereas TC stimulation by supra-threshold stimulation does not activate all of them. The difference in baseline amplitudes between TE-MEP and TC-MEP supports this consideration, although the difference was not statistically significant. Therefore, TE stimulation may be advantageous not only to avoid amplitude fading that is due to change in alpha motor neuron excitability but also to reflect spinal cord function more correctly and promptly than TC stimulation, because the latter may not reflect the function of alpha motor neurons with high firing threshold.

The second and probably more important mechanism may be its sensitivity to spinal tract function. Unlike the time to amplitude reduction/recovery that is influenced by the baseline amplitudes, time to MEP disappearance is not likely to depend on the number of stimulated neurons, and mechanisms other than the stimulation condition may be involved. Lips and colleagues ${ }^{13}$ demonstrated that severe lumber spinal cord ischemia resulted in a rapid loss of myogenic TC-MEP, whereas thoracic spinal cord ischemia of a similar degree resulted in a relatively slow loss of TC-MEP. They explained this result by the difference in vulnerability to ischemia between the synaptic (lumbar anterior horn) and the axonal (thoracic white matter) transmission. This result is consistent with slow disappearance of TC-MEP in dog 6 with spastic paraplegia, in which pathologic examination revealed predominant thoracic spinal tract injury with minimum motor neuron damage at the lumbar spinal cord. Even in this dog, however, time to disappearance of TE-MEP at hindlimbs was quick, which suggests that spinal tract ischemia is quickly reflected to TE-MEP for some reason. Slower return of latency in TC-MEP than in TE-MEP in the 10-minute occlusion group also may support this speculation. Because ischemic injury of the spinal cord is mostly due to combined dysfunction of axons and neurons, sensitivity of TE-MEP to axonal transmission seems to play a role in its quicker response to spinal cord ischemia.

At the anal sphincters, on the other hand, the response time to ischemia was not different between TE-MEP and TC-MEP. Because the absolute number of muscle fibers is considerably smaller in anal sphincters than in hindlimbs, baseline amplitudes were very small, and their change was thus subjected to the influence of electrical noise. In addition, plug-type surface electrodes are more vulnerable to artifacts that are generated by other striated muscles and are predisposed to misalignment from initial position. ${ }^{14}$ Therefore, the use of percentage decrease in amplitude may not be meaningful as an index of spinal cord dysfunction. As far as the time to disappearance is concerned, however, it is shorter in TE-MEP than in TC-MEP, just like those recorded at hindlimbs. Therefore, loss of MEP should be used as an index of spinal cord ischemia recorded at anal sphincters, which will be discussed in the section of its prognostic value. 
The present results suggest that loss of TE-MEP at the hindlimbs without recovery is associated with neurologic deficit, whereas its recovery does not always mean prevention. This is in accordance with the general agreements on the prognostic value of TC-MEP. ${ }^{12}$ However, incomplete paralysis may be predicted by the delay (>30 minutes) in recovery $(>75 \%)$ of hindlimb TEMEPs with persistent loss of anal sphincter TE-MEPs. In this regard, TC-MEP was not reliable because specificity of the persistent loss of anal sphincter potentials in predicting neurologic injury was $63 \%$, whereas it was $100 \%(5 / 5)$ by TE-MEP.

TE-MEP may be advantageous in predicting spastic paraplegia, which could not be detected by TC-MEP in the present study. Spastic paraplegia may result from the injury of upper motor neurons without lower motor neuron injury, a situation observed in dog 6. Although spastic paraplegia seems rare in clinical spinal cord ischemia, Kakinohana and colleagues ${ }^{15}$ reported 1 such case. They also showed in a rat aortic occlusion model that TC-MEP could be normal in animals with spastic paraplegia, which was caused by damage in the spinal cord interneurons. ${ }^{15}$ In normal motor function, tonic activity of excitation and inhibition are balanced mutually, and inhibition is mediated through the interneurons located in the gray matter. ${ }^{16}$ Injury in the spinal cord interneurons thus can cause spastic paraplegia without injury in the pyramidal tract. Although this is an extreme situation, partial injury of the thoracic spinal tract may cause a similar situation, with TC-MEP remaining almost intact.

The eventual goal of this experimental series is clinical application. We have previously shown that TE-MEP is free of the risk of esophageal injury or cardiac rhythm disturbance, its setting is easy and stable, and it can be recorded with a conventional equipment for TC-MEP. ${ }^{9}$ With the present results, we think that we are ready to proceed to the clinical application once the remaining problem of strenuous forelimb movement can be solved. Because this movement is mediated through direct stimulation of the cervical alpha motor neurons, it is not seen in the hindlimbs that are mediated through synaptic transmission. However, this makes recording from the forelimbs unsuitable for real-time control. We are now conducting an experiment to solve this final problem with promising results and expect that TE-MEP will soon be applicable in the clinical settings.

\section{Study Limitations}

For aortic occlusion, we used only 2 time intervals at a fixed aortic level, and all animals in the 10-minute group showed recovery, whereas the majority were paraplegic in the 40-minute group. Although this protocol was sufficient to show that TE-MEP can respond to spinal cord ischemia and timely reperfusion as sensitively as and more quickly than TC-MEP, its advantage in the prognostic value was not conclusive. In this regard, addition of an intermediate time interval of aortic occlusion or occlusion at a higher aortic level might provide more insight into the utility of TE-MEP.

\section{CONCLUSIONS}

TE-MEP seems superior to TC-MEP in terms of quicker response to spinal cord ischemia and better prognostic value.

\section{Conflict of Interest Statement}

Authors have nothing to disclose with regard to commercial support.

You can watch a Webcast of this AATS meeting presentation by going to: http://webcast.aats.org/2015/Video/ Tuesday/04-28-15_6B_0720_Tsuda.mp4.

The authors thank Toshihiko Hasegawa for invaluable technical assistance and Jun Furuya for statistical advice.

\section{References}

1. de Haan P, Kalkman CJ, de Mol BA, Ubags LH, Veldman DJ, Jacobs MJ. Efficacy of transcranial motor-evoked myogenic potentials to detect spinal cord ischemia during operations for thoracoabdominal aneurysms. J Thorac Cardiovasc Surg. 1997;113:87-100.

2. Griepp RB, Griepp EB. Spinal cord protection in surgical and endovascular repair of thoracoabdominal aortic disease. J Thorac Cardiovasc Surg. 2015; 149:S86-90.

3. Meylaerts SA, Jacobs MJ, van Iterson V, De Haan P, Kalkman CJ. Comparison of transcranial motor evoked potentials and somatosensory evoked potentials during thoracoabdominal aortic aneurysm repair. Ann Surg. 1999; 230:742-9.

4. Sloan TB, Heyer EJ. Anesthesia for intraoperative neurophysiologic monitoring of the spinal cord. J Clin Neurophysiol. 2002;19:430-43.

5. Lips J, de Haan P, de Jager SW, Vanicky I, Jacobs MJ, Kalkman CJ. The role of transcranial motor evoked potentials in predicting neurologic and histopathologic outcome after experimental spinal cord ischemia. Anesthesiology. 2002;97: 183-91.

6. Kawanishi Y, Munakata H, Matsumori M, Tanaka H, Yamashita T, Nakagiri K, et al. Usefulness of transcranial motor evoked potentials during thoracoabdominal aortic surgery. Ann Thorac Surg. 2007;83:456-61.

7. van Dongen EP, Schepens MA, Morshuis WJ, ter Beek HT, Aarts LP, de Boer A, et al. Thoracic and thoracoabdominal aortic aneurysm repair: use of evoked potential monitoring in 118 patients. J Vasc Surg. 2001;34:1035-40.

8. Jacobs MJ, Mess W, Mochtar B, Nijenhuis RJ, Statius van Eps RG, Schurink GW. The value of motor evoked potentials in reducing paraplegia during thoracoabdominal aneurysm repair. J Vasc Surg. 2006;43:239-46.

9. Tsuda K, Shiiya N, Takahashi D, Ohkura K, Yamashita K, Kando Y. Transoesophageal spinal cord stimulation for motor-evoked potentials monitoring: feasibility, safety and stability $\dagger$. Eur J Cardiothorac Surg. 2015; 48:245-51.

10. Mochida K, Shinomiya K, Komori H, Furuya K. A new method of multisegment motor pathway monitoring using muscle potentials after train spinal stimulation. Spine. 1995;20:2240-6.

11. Sugawara Y, Kumagai H, Sueda T. A novel canine model of spinal cord ischemia with reproducible neurologic outcomes. Surg Today. 2005;35:649-52.

12. Macdonald DB. Intraoperative motor evoked potential monitoring: overview and update. J Clin Monit Comput. 2006;20:347-77.

13. Lips J, de Haan P, Bouma GJ, Jacobs MJ, Kalkman CJ. Delayed detection of motor pathway dysfunction after selective reduction of thoracic spinal cord blood flow in pigs. J Thorac Cardiovasc Surg. 2002;123:531-8. 
14. Wiesner A, Jost WH. EMG of the external anal sphincter: needle is superior to surface electrode. Dis Colon Rectum. 2000;43:116-8.

15. Kakinohana M, Kawabata T, Miyata Y, Sugahara K. Myogenic transcranial motor evoked potentials monitoring cannot always predict neurologic outcome after spinal cord ischemia in rats. J Thorac Cardiovasc Surg. 2005;129:46-52.

16. Beneck R. Basic neurophysiological mechanisms in spasticity. In: Marsden CD, ed. Treating Spasticity: Pharmacological Advances. Toronto: Hans Huber Publishers; 1989:11-9.

Key Words: aortic surgery, spinal cord ischemia, motor evoked potentials

\section{Discussion}

Dr T. Reece (Denver, Colo). I'm a big proponent of neuromonitoring in the thoracic aortic intervention, but it makes me incredibly frustrated with the number of false-positives in this monitoring system that we use today. I continue to defend the use of neuromonitoring in my cases, but it's hard to argue with the fact that we're overtreating patients because we lose evoked potentials on one side or the other or they're probably not real in terms of the injury. I think there is a role for early aggressive treatment of these patients if we can identify it, but we need a better way to identify it reliably.

In this study, you compared traditional TC evoked potentials with the innovative TE evoked potentials. Comparing these approaches, you demonstrated earlier loss of waveforms and earlier recovery with the TE approach. This correlated closely with the recovery of the predicted dense paraplegia. I applaud your neuromonitoring team, and I would love to have the team you have there come to my operating room because it seems more reliable than what we're doing.

What is the difference in the stimulation that you're giving? When I talked to our neuromonitoring team, the higher the voltage we give the more reliable it probably is. Are you able to change that with the TE approach? I just didn't see a lot of it in the methodology. Why is it different? Why is the spinal cord responding more reliably in this fashion?
Dr Tsuda. We previously reported ${ }^{9}$ that TE stimulation can be performed by supra-maximal intensity, whereas TC stimulation intensity is usually supra-threshold that does not elicit a maximum response. There are 2 reasons: The first is concern about postoperative convulsive seizures due to high-intensity electrical stimulation, on head stimulation. The second is limitation of the equipment we used. The maximum electrode power of our equipment was limited to 600 volts.

Dr Reece. I think that needs to be played out more in the article for us to really understand the differences there. When you do these TE, you're going to lose the ability to compare with the upper extremities, which is one way that we're able to decide if there is an injury, if there are problems with occlusion from our large sheath in the femoral artery, those types of things. Do you think there is any way that we can work on comparing that with the upper extremities or is this something that would be limited to only the lower extremities, the hind limb, above potentials with your method?

Dr Tsuda. I'm sorry, I did not follow your question. What do you mean?

Dr Reece. When we use evoked potentials, we're usually comparing the evoked potential amplitude in the upper limbs compared with the lower limbs. That has become a more reliable way for us to understand if that amplitude is gone. In this study it sounds like getting upper-extremity amplitudes is not possible with the esophageal probe. Is that something that would be possible or is that lost by going further down in the chest with your stimulation?

Dr Tsuda. As you pointed out, upper-limb amplitudes were unsuitable for real-time control because they were elicited by direct stimulation of the forelimb alpha motor neurons and not through synaptic transmission. ${ }^{9}$ We are now trying a modified stimulation technique with a deeper esophageal probe position to solve this problem. The results are encouraging. 